\title{
INDEX OF FREDHOLM OPERATORS
}

\author{
KUNG-WEI YANG
}

Abstract. Let $X, Y, Z$ be Banach spaces, and let $T: X \rightarrow Y$ and $S: Y \rightarrow Z$ be Fredholm operators. Let ind $(T)$ denote the index of $T$. A short proof is given for the identity ind $(S T)=\operatorname{ind}(S)+\operatorname{ind}(T)$.

We give a "one-diagram" proof of the following theorem $[3$, Theorem 3, p. 121]. The notation of [3] will be used.

THEOREM. Let $X, Y, Z$ be Banach spaces, and $T: X \rightarrow Y$ and $S: Y \rightarrow Z$ be Fredholm operators. Then

$$
\operatorname{ind}(S T)=\operatorname{ind}(S)+\operatorname{ind}(T) .
$$

Proof. By [3, Corollary 3, p. 121], $S T$ is a Fredholm operator. It is easy to verify directly that the outer perimeter sequence in the following commutative diagram of Banach spaces is exact $[1$, p. 25].

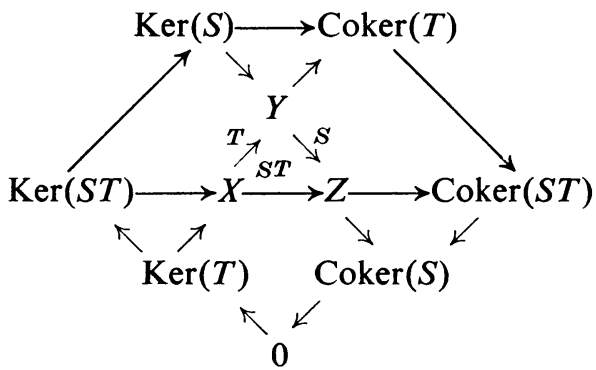

Now notice that if $0 \rightarrow A_{0} \rightarrow A_{1} \rightarrow \cdots \rightarrow A_{n} \rightarrow 0$ is an exact sequence of finite dimensional Banach spaces, then

$$
\sum_{i=0}^{n}(-1)^{i} \operatorname{dim}\left(A_{i}\right)=0 .
$$

The desired equation follows immediately.

Corollary 1. Let $F: X \rightarrow X$ be a Fredholm operator. Then, if for $n \geqq 0$, ind $\left(T^{n}\right)=0$, then ind $(T)=0$.

Received by the editors March 2, 1973.

AMS (MOS) subject classifications (1970). Primary 47B30.

Key words and phrases. Index of Fredholm operator.

(c) American Mathematical Society 1973 
Corollary 2. Let $K: X \rightarrow X$ be a compact operator, and $I: X \rightarrow X$ be the identity operator. Then, $\operatorname{ind}(I+K)=0$.

Proof. This follows from [2, (11.3.3), p. 321] and Corollary 1.

\section{BIBLIOGRAPHY}

1. Hyman Bass, Algebraic K-theory, Benjamin, New York, 1968. MR 40 \#2736.

2. J. Dieudonné, Foundations of modern analysis, Academic Press, New York and London, 1969.

3. Richard S. Palais, Seminar on the Atiyah-Singer index theorem, Ann. of Math. Studies, no. 57, Princeton Univ. Press, Princeton, N.J., 1965. MR 33 \#6649.

Department of Mathematics, Western Michigan University, Kalamazoo, MiCHIGAN 49001 